\title{
Anaemia prevalence and factors associated with haemoglobin change following radiotherapy in individuals with cancers at the Ocean Road Cancer Institute in Tanzania
}

\author{
ABEL MAKUBI*1,2, ANDREW SHIYO ${ }^{1}$ and CRISPIN KAHESA ${ }^{3}$

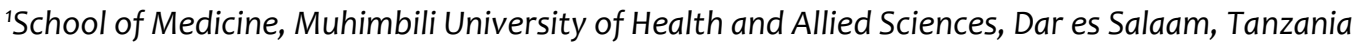 \\ 2Department of Medicine, Muhimbili National Hospital, Dar es Salaam, Tanzania \\ ${ }^{3}$ Department of Oncology, Ocean Road Cancer Institute, Dar es Salaam, Tanzania
}

\begin{abstract}
Background: Despite the fact that anaemia is common worldwide, limited studies have been done in most developing countries to estimate its prevalence and factors associated with haemoglobin change in patients with cancers receiving radiotherapy.

Methods: This was a descriptive cross-sectional study conducted at the Ocean Road Cancer Institute in Dar es salaam, Tanzania. All individuals with cancers who were admitted in the wards for radiation treatment from August to December 2013 were included. Information on social-clinical characteristics, cancer type and associated factors as well as haemoglobin level before and after radiation were obtained. The prevalence of anaemia was determined as a proportion and linear regression was used to determine factors associated with haemoglobin change.

Results: A total of 230 study subjects were available for analysis, of whom $82 \%$ were females. The overall mean age was $50 \pm 14$ years. Most of the subjects (44\%) were residing in the coastal regions, $50 \%$ had never attended school and $77 \%$ had no formal employment. The overall prevalence of anaemia was $77 \%$ before and $82 \%$ after radiotherapy. Multivariate linear regression analysis revealed that history of blood transfusion (beta-coefficient $=1.193 ; p=0.004$ ) was the only factor associated with absolute change in haemoglobin.

Conclusion: The prevalence of anaemia was high, regardless of the cancer type. Blood transfusion appeared to have a positive change in haemoglobin following radiotherapy. Awareness should be increased to provide early detection of the condition with appropriate corrective measures.
\end{abstract}

Keywords: cancer, anaemia, haemoglobin, radiotherapy, Tanzania

\section{Introduction}

Anaemia is a common and significant public health problem worldwide and its magnitude has been extensively studied in developed countries. It has been identified in $9 \%$ to $90 \%$ of patients who have been diagnosed to have cancer, depending on the type of cancer (Balducci, 2003; Knight et al., 2004). In Sub-Saharan Africa (SSA), the burden of anaemia in this unique population is less well known. Anaemia results in poorer survival and slow local tumour regression (Obralić et al., 1990) The most serious complication of anaemia arise from tissue hypoxia which is associated with poor treatment outcome after radiotherapy (Glaspy \& Cavill, 1999)

Association between anaemia and radiotherapy in patients with cancers has been well established in developed countries (Harrison et al., 2000, 2002; Serkies et al., 2006). In a series of studies, Harrison et al. $(2000,2002)$ found the prevalence of anaemia in patients with cancers to be $9-55 \%$ before radiotherapy and $57-82 \%$ after radiotherapy. Awareness of this burden in developed countries has helped to reduce the incidence and treatment burden in their settings, resulting into significantly low morbidity and mortality related to cancer (Harrison et al., 2000; Shasha \& Harrison, 2001; Knight et al., 2004). Limited statistics of anaemia compounded with few medical facilities might result in poor management and outcome of these patients in Tanzania.

Factors associated with negative or positive haemoglobin changes following radiotherapy, are not clearly known, a better understanding of which might draw attention to the public health authorities on the need to re-evaluate current strategies to control anaemia by making sure that the contributing factors are identified and addressed properly. Thus the current study aimed to 
determine the prevalence of anaemia and factors associated with haemoglobin change following radiotherapy in individuals with cancers at Ocean Road Cancer Institute in Tanzania.

\section{Materials and Methods}

\section{Study design and setting}

This was a descriptive cross sectional study of individuals with cancers who were admitted at Ocean Road Cancer Institute (ORCI) for radiation therapy from August to December 2013. Ocean Road Cancer Institute is the only health institution for cancer treatment in Tanzania in Dar es Salaam. It offers laboratory services, diagnostic imaging, chemotherapy, radiotherapy and palliative care services. The institute has a total of 257 beds, and approximately 30 patients are admitted per day.

\section{Data collection}

All consecutive patients aged $\geq 18$ years, diagnosed with cancer and admitted for radiotherapy were included in this study. Those who did not provide consent for participation into the study were excluded. Patient's demographic data (age sex, residence, education and occupation), medical history on when the disease was diagnosed, full blood picture results, radiation history on the dose of radiation received, history of chemotherapy, blood transfusion, and use of haematinic during radiation therapy were obtained from hospital medical records. Residence was grouped into zones: Coastal Zone (Tanga, Morogoro, Dar es Salaam, and Zanzibar); Northern Highlands (Arusha and Kilimanjaro); Lake Zone (Tabora, Kigoma, Shinyanga, Kagera, Mwanza and Mara); Central Zone (Dodoma and Singida); Southern Zone (Iringa, Mbeya, Rukwa, Lindi, Mtwara and Ruvuma). This information was recorded in the checklist prepared and later analysed.

\section{Blood sample analysis}

Blood samples were used to generate full blood count measures (including haemoglobin) from each admitted patient. Venous blood sample was obtained in a closed blood collection system (Monovette) with EDTA, and later processed at the central laboratory of OCRI. Full blood pictures were obtained by means of a haematology analyser (Beckman Coulter ACT5 DIFF) and were collected within one week, before and after radiotherapy.

\section{Data analysis}

Data was entered into a computer and cleared using SPSS package software version 20 . The prevalence of anaemia before and after radiation was determined as a percentage among all admitted cancer patients whose haemoglobin level were below the WHO cut-offs based on sex. Anaemia being the primary outcome was defined by WHO as reduced concentration of haemoglobin in blood below $13.0 \mathrm{~g} / \mathrm{dl}$ for males and $12 \mathrm{~g} / \mathrm{dl}$ for females. The secondary outcome was haemoglobin change (in $\mathrm{g} / \mathrm{dl}$ ) calculated as a difference between haemoglobin levels before and after radiotherapy. Linear regression was used to determine factors associated with haemoglobin change following radiotherapy. All analysis was conducted with SPSS version 20, and the test of significance was evaluated with a probability cut-off value of 0.05 .

\section{Ethical considerations}

The study and consent procedures were approved by the Muhimbili University of Health and Allied Sciences Ethical Review Board. Permission to conduct the study was obtained from the Hospital in-charge of Ocean Road Cancer Institute. Prior informed written consent (in the local language (Kiswahili) was sought from all patients who had to agree or disagree with study participation and provided a documented signature. Patients who were diagnosed to have anaemia were treated accordingly either by haematinics or blood transfusion depending on the severity of the problem. 


\section{Results}

\section{Characteristics of study patients}

A total of 275 patients were recruited in the study of whom 45 were excluded because their data were incomplete or died during the study period (Figure 1).

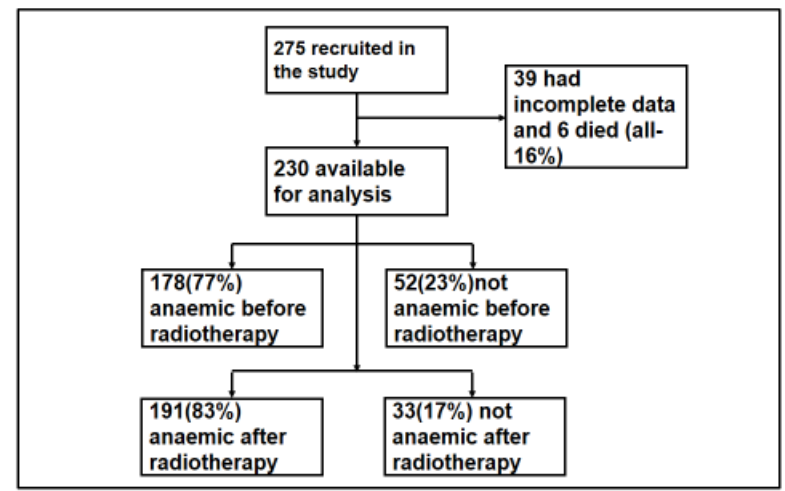

Figure 1. Flow chat for patients included for analysis

Table 1: Socio-demographic and clinical characteristics of patients with cancers by sex

\begin{tabular}{llllllll}
\hline Variable & Response & All & \multicolumn{3}{c}{ Male } & \multicolumn{2}{l}{ Female } \\
& & $\mathbf{N}$ & $\%$ & $\mathbf{N}$ & $\%$ & $\mathbf{N}$ & $\%$ \\
\hline Age group (years) & $<35$ & 30 & 13 & 14 & 33.3 & 16 & 8.5 \\
& $36-50$ & 110 & 47.8 & 21 & 50 & 89 & 47.3 \\
\multirow{3}{*}{ Residence } & $>51$ & 90 & 39.1 & 7 & 16.7 & 83 & 44.1 \\
& Northern Highlands & 17 & 7.4 & 5 & 11.9 & 12 & 6.4 \\
& Coastal Zone & 100 & 43.5 & 18 & 42.9 & 82 & 43.6 \\
& Lake Zone & 31 & 13.5 & 3 & 7.1 & 28 & 14.9 \\
& Southern Highlands & 39 & 17 & 8 & 19 & 31 & 16.5 \\
Education & Central Zone & 43 & 18.7 & 8 & 19 & 35 & 18.6 \\
& None & 115 & 50 & 16 & 38.1 & 99 & 52.7 \\
& Primary & 105 & 45.7 & 23 & 54.8 & 82 & 43.6 \\
Occupation & Secondary & 8 & 3.5 & 1 & 2.4 & 7 & 3.7 \\
& College & 2 & 0.9 & 2 & 4.8 & 0 & 0 \\
& Unemployment & 45 & 19.6 & 3 & 7.1 & 42 & 22.3 \\
Cancer type & Informal & 178 & 77.4 & 37 & 88.1 & 141 & 75 \\
& Formal & 7 & 3 & 2 & 4.8 & 5 & 2.7 \\
& Cervix & & & & & 139 & 73.9 \\
& Breast & & & & & 11 & 5.9 \\
& Oesophagus & 9 & 3.9 & 6 & 14.3 & 2 & 1.1
\end{tabular}

A total of 230 cancer treated patients met the criteria to be included in the study. The study subjects were aged 15 to 92 years with overall mean age of $50 \pm 14$ years (Table 1). Majority of the study subjects (48\%) were in the age group 36-50 years, were females (82\%) and had no formal employment (77\%). Likewise, majority of the patients had cervical cancer $(60 \%)$ followed by breast 
(5\%), oesophageal (4\%), Kaposi Sarcoma (4\%), Colorectal (3\%) and Prostate (0.4\%) cancers. Other types of cancer contributed to $25 \%$ of the patients admitted (Table 1 ).

\section{Prevalence of anaemia in individuals with cancers}

Among all patients who were in the wards during the study period $77 \%$ were anaemic on admission. After (within one week) treatment with the single dose of radiation there was increase to $83 \%$. In males, the prevalence of anaemia before radiation was $81 \%$ and after radiation was $86 \%$, while in females the prevalence before radiation was $77 \%$ and $85 \%$ after radiation (Figure 2). Anaemia in patients with cervical cancer was $78 \%$ before radiation and $87 \%$ after radiation, in breast cancer was $58 \%$ before and $92 \%$ after, in oesophageal cancer was $0.9 \%$ and later dropped to $0.7 \%$ and in colorectal cancer was $100 \%$ and dropped to $50 \%$ after radiotherapy (Table 2 ).
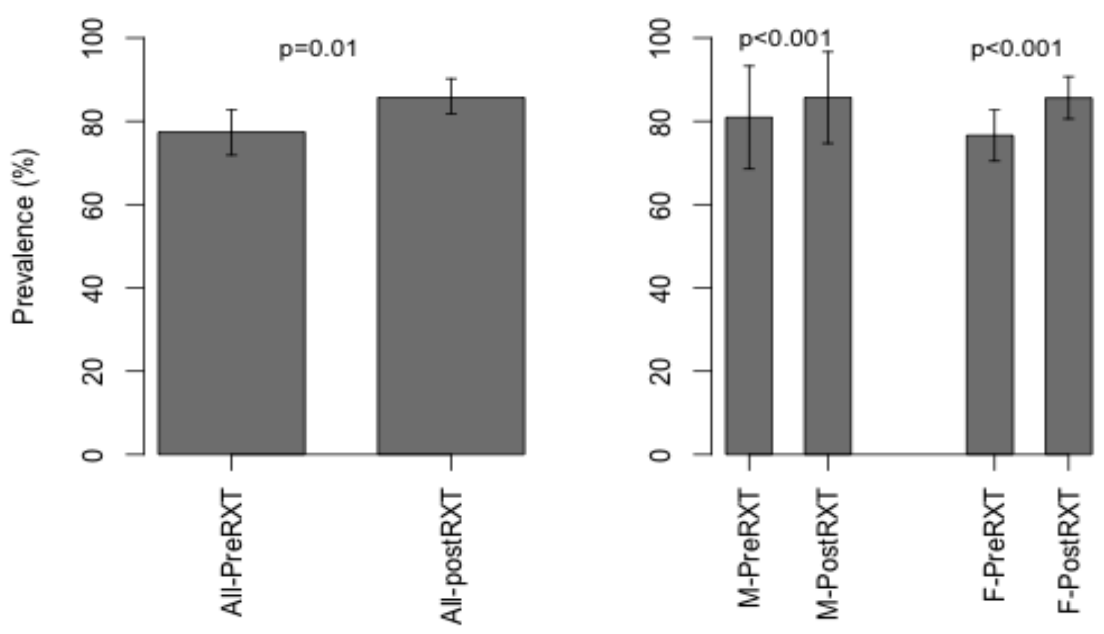

Figure 2: Prevalence of anaemia pre- and post-radiation-therapy in patients with cancers
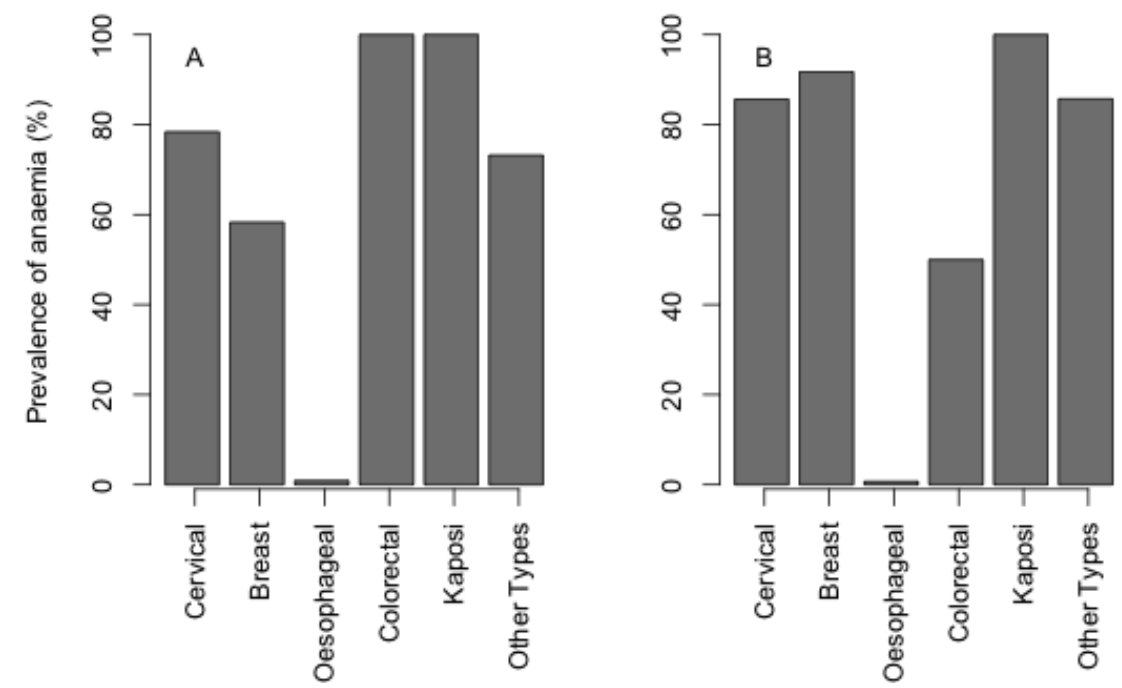

Figure 3: Prevalence of anaemia in various cancer types, before $(A)$ and after (B) radiotherapy

\section{The factors associated with haemoglobin change}

Multivariate linear regression analysis (Figure 2) revealed only one factor associated with absolute change in haemoglobin; history of blood transfusion (beta-coefficient=1.193; $p=0.004$ ). The mean haemoglobin change among patients who received blood transfusion was $1.2 \mathrm{~g} / \mathrm{dl}$ higher than those patients who did not receive. Both in univariate and multivariate linear regression analyses, 
the association between haemoglobin change and age, sex, cancer stage, use of haematinics and use of chemotherapy, did not reach statistical significance.

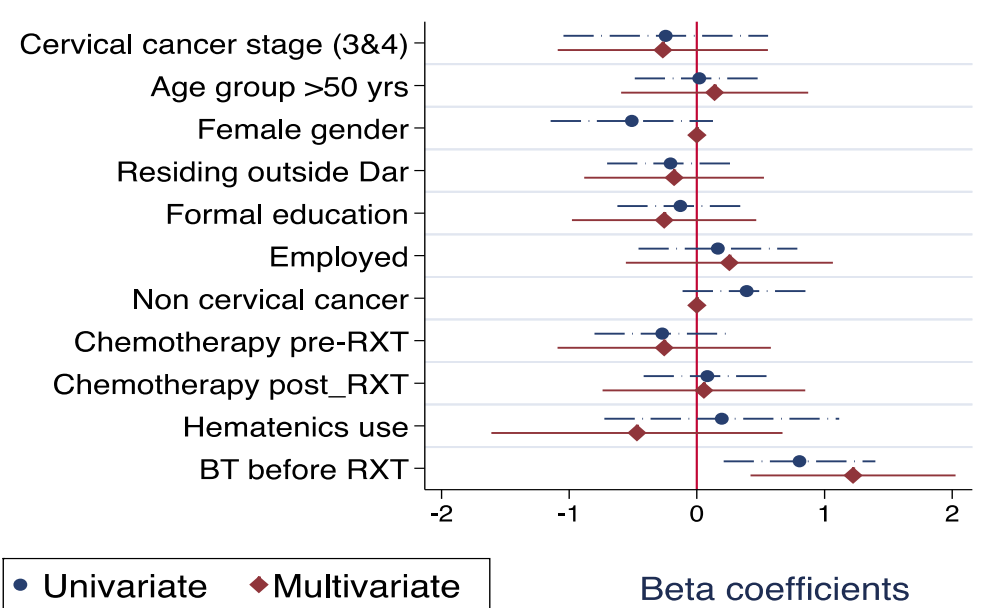

Figure 4: Factors associated with haemoglobin change following radiotherapy in patients with cancers.

\section{Discussion}

This study represents a unique population of individuals with cancers who are able to access radiation therapy in Tanzania. Anaemia was found to be common in this population and increased with exposure to radiotherapy suggesting intervention for improved prognosis. Blood transfusion given before radiation was associated with a positive haemoglobin change. Many observational studies have focused on impact of anaemia on prognosis rather than its prevalence among patients undergoing radiotherapy (Harrison et al., 2000, 2002; Shasha \& Harrison, 2001). Little attention is paid to the dynamics of anaemia prevalence before or after radiotherapy. In the current study, the prevalence of anaemia study was high both before and after radiotherapy but is closely comparable to the findings reported by Harrison et al. (2002) in which the prevalence rose from $78 \%$ before radiation to $86 \%$ after radiation therapy. This implies that within this short duration the level of haemoglobin decreases significantly, restoration of which has the potential to improve local tumour control and survival and produce improvement in quality of life (Harrison et al., 2000). Thus, in Tanzania where anaemia is already prevalent in the general population(Kitange et al., 1993; Makubi et al., 2013), early detection and intervention at this stage is of clinical relevancy.

Interesting, in our study, all patients with colorectal cancer were anaemic before radiation therapy and later after radiation treatment the prevalence dropped by half. This differs from a study by Harrison et al. (2002) which found that $44 \%$ patients with colorectal cancer had anaemia before radiation therapy, and the prevalence rose to $63 \%$ afterward. This difference can be explained by the fact that the number of study subjects with colorectal carcinoma was small (two patients) and also among them one patient received blood transfusion during radiation therapy thus restoring the anaemic status.

Since cancer or treatment-related anaemia is present in up to $90 \%$ of patients, our finding has considerable significance at the population level. Moreover, because haemoglobin level is frequently measured in the routinely tested complete blood count panel, haemoglobin change may be a potentially important variable that can be incorporated with other host and clinical factors to build cancer prognosis assessment models.

The mechanism of anaemia during radiotherapy might be related the direct toxicity to any cells in the body that divide rapidly, which include blood cells and bone marrow cells that make the blood cells. Inflammation caused by irradiation exposure might be another explanation(Grellier et al., 2015). However, the present data do not permit further elaboration on this issue. 
The present findings of association between blood transfusion and positive haemoglobin change have important prognostic and policy implications. Low haemoglobin during radiotherapy leads to tissue hypoxia and has been associated with poor treatment outcome afterwards (Obralić et al., 1990; Glaspy \& Cavill, 1999; Harrison et al., 2002; Wan et al., 2013). A few studies have reported maintaining patient haemoglobin levels $>12.5 \mathrm{~g} / \mathrm{L}$ significantly improves local tumour control rates compared with the rates in patients with lower haemoglobin levels in patients with cervical cancer (Bergsjo, 1965; Bush et al., 1978). Thus blood transfusion centres should provide adequate blood to patients with cancers before and after radiotherapy to counteract the effect of haemoglobin reduction and hence improve the survival of these patients.

Our study has some limitations. The sample size was small and the study was conducted in a very specialized facility and thus might not represent the cancer population in Tanzania. This might have also resulted in lack of statistical power for detection of some associations. Even with these limitations, this study serves as a reference for future recommendations to improve care of patients with cancers by active screening and correction of anaemia during radiotherapy.

In conclusion, we have observed a high prevalence of anaemia in individuals with cancer receiving radiotherapy and only blood transfusion might have a positive effect on haemoglobin dynamics. Therefore, there should be an increase in awareness of this problem to provide early detection of the disease and improve the outcome and quality of life of the patients.

\section{Acknowledgments}

We thank the patients and their families who participated in these studies. The authors also thank Muhimbili University of Health and Allied Sciences and ORCI staff for collaboration in implementing this study. This study received financial support from Muhimbili University of Health and Allied Sciences

\section{Author contributions}

All authors have made significant contributions to the design, execution, analysis and writing of this study

\section{Competing interests}

None

\section{References}

Balducci, L. (2003) Anemia, cancer, and aging. Cancer Control Journal of the Moffitt Cancer Center 10, 478-486.

Bush, R.S., Jenkin, R.D., Allt, W.E., Beale, F.A., Bean, H., Dembo, A.J. \& Pringle, J.F. (1978) Definitive evidence for hypoxic cells influencing cure in cancer therapy. British Journal of Cancer 3, 302306.

Evans, J.C. \& Bergsjo, P. (1965) The influence of anemia on the results of radiotherapy in carcinoma of the cervix. Radiology 84, 709-717.

Glaspy, J. \& Cavill, I. (1999) Role of iron in optimizing responses of anemic cancer patients to erythropoietin. Oncology 13, 461-473.

Grellier, N., Deray, G., Yousfi, A., Khodari, W., Bouaita, R. \& Belkacemi, Y. (2015) Functional iron deficiency, inflammation and fatigue after radiotherapy. Bulletin du Cancer 102, 780-785.

Harrison, L.B., Shasha, D., White, C. \& Ramdeen, B. (2000) Radiotherapy-associated anemia: the scope of the problem. The oncologist 5, 1-7. 
Harrison, L.B., Shasha, D. \& Homel, P. (2002) Prevalence of anemia in cancer patients undergoing radiotherapy: prognostic significance and treatment. Oncology 63, 11-18.

Kitange, H. et al., (1993) Anaemia is a major public health problem in Tanzania. Health Policy and Planning 8, 413-418.

Knight, K., Wade, S. \& Balducci, L. (2004) Prevalence and outcomes of anemia in cancer: a systematic review of the literature. American Journal of Medicine 116, 11-26.

Makubi, A., Okuma, J., Spiegelman, D., Hawkins, C., Darling, A.M., Jackson, E., Mugusi, F., Chalamilla, G. \& Fawzi, W. (2013) Burden and determinants of severe anemia among HIVinfected adults: results from a large urban HIV program in Tanzania, East Africa. Journal of the International Association of Providers of AIDS Care 14, 148-55.

Obralić, N., Bilenjki, D. \& Bilbija, Z. (1990) Prognostic importance of anemia related parameters in patients with carcinoma of the cervix uteri. Acta Oncologica 29, 199-201.

Serkies, K., Badzio, A. \& Jassem, J. (2006) Clinical relevance of hemoglobin level in cervical cancer patients administered definitive radiotherapy. Acta Oncologica 45, 695-701.

Shasha, D. \& Harrison, L.B. (2001) Anemia treatment and the radiation oncologist: optimizing patient outcomes. Oncology 15, 1486-1491.

Wan, S., Lai, Y., Myers, R.E., Li, B., Palazzo, J.P., Burkart, A.L., Chen, G., Xing, J. \& Yang, H. (2013) Post-diagnosis hemoglobin change associates with overall survival of multiple malignancies results from a 14-year hospital-based cohort of lung, breast, colorectal, and liver cancers. BMC Cancer 13, 340. 